\title{
Uso de sistemas de gestión de relaciones con clientes: evidencias de Instituciones de Educación Superior en Colombia*
}

\section{Use of customer relationship management systems: Evidence from Higher Education Institutions in Colombia}

\section{Sistemas utilizando customer relationship management: Evidências de Instituições de Ensino Superior na Colômbia}

\author{
Luis Guillermo Castellanos-Camargo \\ Administrador de Empresas y Magíster en Gerencia Estratégica de Mercadeo. Director de Admisiones, Escuela de Diseño y Mercadeo \\ de Moda Arturo Tejada Cano. \\ admisiones@arturotejada.edu.co
}

\section{Sandra Patricia Rojas-Berrío}

Administradora de Empresas, Magíster en Administración y Doctora en Ciencias Administrativas. Profesor a Asistente - Facultad de Ciencias Económicas, Universidad Nacional de Colombia - Sede Bogotá. sprojasb@unal.edu.co

\section{Óscar Javier Robayo-Pinzón}

Psicólogo y Magíster en Psicología del Consumidor. Profesor Asistente, Facultad de Mercadeo, Comunicación y Artes, Institución Universitaria Politécnico Grancolombiano.

osrobayo@poligran.edu.co

\section{Resumen}

Este documento explora el uso de Sistemas de Gestión de Relaciones con Clientes (CRM) en Instituciones de Educación Superior (IES) en Colombia. Se realizaron ocho entrevistas en profundidad a los gestores de las acciones de mercadeo en IES privadas, la información fue procesada en NVIVO. Los hallazgos sugieren que las IES entrevistadas usan CRM; sin embargo, algunas realizan campañas de mercadeo a través de otros canales (por ejemplo: otros sistemas de información o sin registro en algún sistema). Lo anterior lleva a la desarticulación y al reproceso de actividades de mercadeo y tiene como implicación gerencial la necesidad de generar mecanismos que permitan que la informacion y la gestión

\footnotetext{
* Cómo citar: Castellanos-Camargo, L. G., Rojas-Berrío, S.P., Robayo-Pinzón, 0. J. (2017). Uso de sistemas de gestión de relaciones con clientes: evidencias de Instituciones de Educación Superior en Colombia. Revista Libre Empresa, 14(1), 241-252 http://dx.doi. org/10.18041/libemp.2017.v14n1.27111
} 
de las estrategias de mercadeo conformen la cultura de gestión de conocimiento de las IES, con el fin de mantener y hacer seguimiento a los estudiantes a través de todo el potencial con el que cuenta el CRM, máxime en un país con altas tasas de deserción.

\section{Palabras clave}

Gestión de relaciones con los clientes; Instituciones de educación superior; salida del estudiante.

\section{Abstract}

This document explores Customer Relationship Management Systems (CRM) use at Higher Education Institutions (HEI's), in Colombia. In-depth interviews were conducted involving 8 marketing managers at private HEI's, information was processed in NVIVO. Findings indicate that all HEI's interviewed use CRM; however, some conduct campaigns through other sources (i.e. information systems or manually), which leads to further disarticulation of marketing activities, due to reprocessing. The above has as managerial implication the need to generate mechanisms that allow information and marketing management strategies as part of organization culture, to keep and track customers knowledge through CRM potential, in a country with high levels of student dropout.

\section{Keywords}

Customer relationship management; Higher Education Institutions; Student Dropout.

\section{Resumo}

Este artigo explora o uso de sistemas de Customer Relationship Management (CRM) em Instituições de Ensino Superior (IES), na Colômbia. 8 gerentes entrevistas ações de marketing em profundidade foram realizadas IES privadas, as informações foram processadas no NVivo. Os resultados sugerem que o uso de CRM IES entrevistados; no entanto, algumas campanhas de marketing realizadas através de outros canais (por exemplo, outros sistemas de informação sem registro ou qualquer outro sistema). Isso leva ao desmantelamento e reprocessamento de atividades de marketing. Isto tem como implicação gerencial a necessidade de criar mecanismos que permitem estratégias de informação e de marketing gestão, conformam a cultura de conhecimento da IES de gestão, a fim de manter e controlar os alunos através todo o potencial que conta CRM, especialmente em um país com altas taxas de evasão.

\section{Palavras chave}

Gerenciar relacionamentos com clientes; instituições de ensino superior; Estudante fora.

\section{Antecedentes}

La gestión del servicio educativo en las Instituciones de Educación Superior (IES) en Colombia, es un tema poco explorado en la literatura. No obstante, diversos documentos se dedican a resaltar textos como la gestión educativa, la calidad de la formación, los planes de educación anual, el diseño de currículos y las metas en el corto plazo, pero ninguno se 
centra en cómo se usa o se han apropiado sistemas de información para gestión de relaciones con los estudiantes, desde una perspectiva de marketing, si bien existen estudios previos que han realizado mediciones puntuales de la satisfacción de los estudiantes (MontoyaRestrepo, Montoya-Restrepo, \& Rojas-Berrío, 2012).

En el campo de los servicios que prestan las IES a los estudiantes, Seeman y O'Hara (2006) muestran cómo la satisfacción con los programas y servicios de una universidad es también una medida de rendimiento crítica, entonces, la Gestión de Relaciones con Clientes cobra un rol importante, dado que los estudiantes no sólo esperan proceso de aprendizaje significativos sino una experiencia universitaria en términos de servicio. En este sentido, un sistema de información CRM podría llegar a optimizar las actividades de marketing en una IES como: personalización de comunicaciones para aspirantes, selección de IES y fomento a la permanencia (Seeman \& O’Hara, 2006).

Esta investigación acoge como ámbito a Colombia, dado que el abandono estudiantil en Educación Superior para 2013 alcanzó un límite de alrededor del 44.9 \%, según la más reciente medición del Ministerio de Educación, lo que implica que uno de cada dos estudiantes matriculados no completa sus estudios; aunado a lo anterior, un estudio previo en este país muestra que las fallas organizativas y de gestión son una problemática institucional que influye en la deserción estudiantil (Santamaría \& Bustos, 2013).

Puesto que la literatura indica que el seguimiento al estudiante puede darse por la vía de un CRM (Abubakar \& Mokhtar, 2015; Bejou D., Bejou A, 2012), esta investigación busca determinar si las instituciones de educación superior usan el Customer Relationship Management (CRM) para realizar seguimiento a los estudiantes, y en específico, a la posible deserción.

\section{Revisión de literatura Gestión de relaciones con clientes}

Según Gil La fuente, y Luis-Bassa (2011) las estrategias de relación con los clientes, mejor conocidas como estrategias CRM, colocan al cliente como el centro de la empresa y alinean todo el negocio a su alrededor; así mismo es un programa gradual que requiere una reflexión permanente sobre el modelo estratégico interno, un compromiso constante de todos los integrantes de la compañía y un cambio de mentalidad enfocado al cliente, desde la cabeza hasta la base de la organización (Hernández-Mejía, 2008).

En el marco de lo anterior, es relevante indicar que CRM cuenta con diversos significados en la literatura, pero el objetivo final perseguido por cualquier organización que utilice este tipo de sistemas es proporcionar a sus clientes un valor añadido, basado en la inteligencia individualizada de sus preferencias (Daradoumis et al., 2008). Es así cómo, el interés en la implementación de estos sistemas en las Instituciones de Educación Superior -en adelante IES- ha evolucionado de forma importante (Khashab, Gulliver, Alsoud, \& Kyritsis, 2014).

En el trabajo de Frow, Payne, Wilkinson \& Young (2015) se indica que en Gestión de Relaciones con los Clientes -de aquí en adelante CRM- su propósito y enfoque son 
estratégicos, en tanto que constituyen una forma de gestión orientada a los datos y a la organización enfocada en la creación de valor para los accionistas a través de la mejora y desarrollo de las relaciones adecuadas con los principales clientes y segmentos de clientes. Por tanto, requiere de generar y co-crear valor con los clientes y para tal fin debe existir integración de funciones cruzadas del proceso, las personas, las operaciones y capacidades de marketing a través de la información y, por ende, el uso correcto de la tecnología.

En los sistemas educativos, según Sahney, Banwet, \& Karunes (2008), se pueden identificar dos tipos de cliente: los internos y los externos. En este sentido, como lo anotan Seeman y O’Hara (2006), refiriéndose al sistema estudiantil cuando describen que los componentes del sistema del estudiante incluyen: expediente académico, las cuentas por cobrar en recibos / efectivo, las organizaciones del campus, gestión curricular, auditoría de grado, la información de la facultad, la ayuda financiera, gestión de contratación / admisiones, registro, y la vida en la residencia.

Lo anterior, lo corrobora en parte Bejou y Bejou (2012), cuando anota que una adaptación del modelo de CRM a la educación superior también dará lugar a cuatro fases: 1) el reclutamiento; 2) la gestión de la matrícula; 3) la retención y persistencia; 4) la graduación.

Por tanto, el concepto de CRM no puede ser visto exclusivamente como una herramienta informática o software, sino como una estrategia, y así lo hace notar Petrella (2008), toda vez que afirma que es importante tener presente que la disponibilidad de una solución tecnológica adecuada, solo contempla una parte de los desafíos de cambio que habrá que superar. Así mismo, en este contexto las tecnologías generan cambios en los mecanismos para abordar las relaciones con los estudiantes. Según el autor, tanto las transacciones electrónicas como el Internet como medio para ellas han gestado cambios revolucionarios en todos los sectores, la educación no es ajena a ello.

Es relevante tener en cuenta que los clientes no siempre están satisfechos con lo que reciben, por tanto el CRM no debe pensarse exclusivamente como una herramienta para enfrentar esta situación, sino como una filosofía que permite gestionar cualquier tipo de relación con los clientes. En el caso educativo a este respecto Duževic \& Ceh Casni (2015) identifican las percepciones de la calidad del servicio de estudiantes y profesores, y del papel moderador de los aspectos institucionales, en los que los principios de gestión requieren de cambios culturales y de la estructura organizacional.

Para el contexto educativo, el marketing relacional debe contar con estrategias para atraer, establecer y consolidar relaciones con las partes interesadas, que son los estudiantes, los padres, los grupos de referencia y los organismos gubernamentales pertinentes; con énfasis en la retención de los estudiantes existentes y prospección de clientes potenciales (Abubakar \& Mokhtar, 2015).

Rowley (2003) muestra cómo el abandono de los estudiantes de la Educación Superior suponen importantes costos institucionales y personales, en este escenario es probable que 
las instituciones tengan en cuenta sus procedimientos de admisión, información sobre el curso y la institución, los servicios de asistencia y consejería estudiantil y las finanzas estudiantiles, en un intento por investigar las causas de la no finalización. Así mismo, en las IES existen aspectos susceptibles de ser controlado por parte de la institución utilizando tecnologías CRM, tal como se realiza en otros ámbitos organizacionales (Bejou D., Bejou A, 2012; Gil-Lafuente \& Luis-Bassa, 2011; Pember, Owens, \& Yaghi, 2014).

\section{Estrategia metodológica}

El proyecto se llevó a cabo con un enfoque pragmático, el abordaje de participantes se realizó con métodos cualitativos y muestreo intencional. Se contó con la participación de ocho IES en Colombia, convocadas por la Asociación de Instituciones de Crédito Educativo (APICE).

Es relevante indicar que como mecanismo de inclusión se seleccionaron IES que contaran con sistemas de información formales, que contribuyeran a la gestión de relación con los clientes y que tuvieran gestión y control de las cifras de deserción estudiantil. Como parámetros de confiabilidad se tuvo en cuenta lo indicado por Kornbluh (2015) y la información obtenida de las entrevistas fue procesada en NVivo.

El instrumento se elaboró teniendo en cuenta como ejes de indagación las fases que indica Bejou y Bejou (2012) en el marco de la adaptación de un CRM para el caso de las IES: 1) el reclutamiento; 2) la gestión de la matrícula; 3) la retención y persistencia; 4) la graduación.

\section{Resultados}

La Figura 1 representa los ejes de indagación en los que interviene el CRM en el marco del proceso de formación de un estudiante: reclutamiento, gestión de matrícula, retención y persistencia y graduación. Así mismo, para cada uno de estos nodos, que son los mismos ejes de indagación del instrumento, existen procesos asociados a evitar la deserción de los estudiantes, siendo este un asunto de interés transversal para los cuatro procesos evaluación.

No obstante, desde la visión de los participantes los procesos están desarticulados y existen varias plataformas tecnológicas en las áreas académica, financiera y CRM; además de las páginas web y redes sociales que se utilizan en las IES entrevistadas, todas estas plataformas generan información para las áreas de interés, pero no existen mecanismos claros para articularla y utilizarla en la gestión de estudiantes.

Es así que, el NODO de Reclutamiento representado en la Figura 2 se detectan varios nodos dependientes dentro de la estructura, en lo que se evidencia que uno de los aspectos más relevantes es el significado que se le da al CRM desde la visión de los participantes, que es: "Gestión para relaciones con los clientes” Así, como lo define en esta entrevista, se evidencia que la percepción que se tiene del CRM en este caso no es una filosofía institucional, toda vez que lo más importante es la relación directa con los estudiantes. 


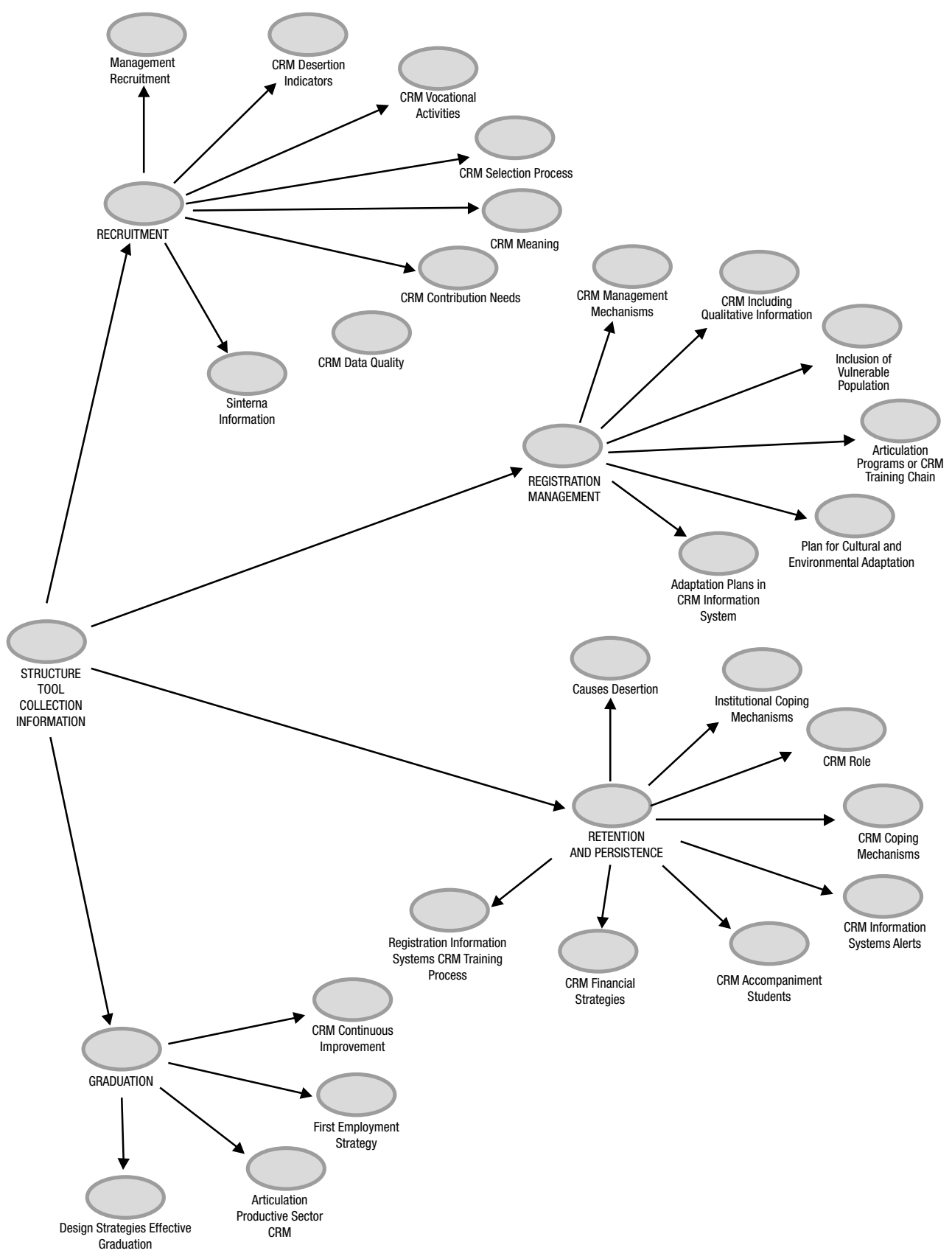

Figura 1. Uso y apropiación de los Sistemas de Información para Gestión de Relaciones con los clientes (CRM) - en IES en Colombia

Fuente: elaboración propia en NVivo 11 a partir de entrevistas semiestructuradas. 


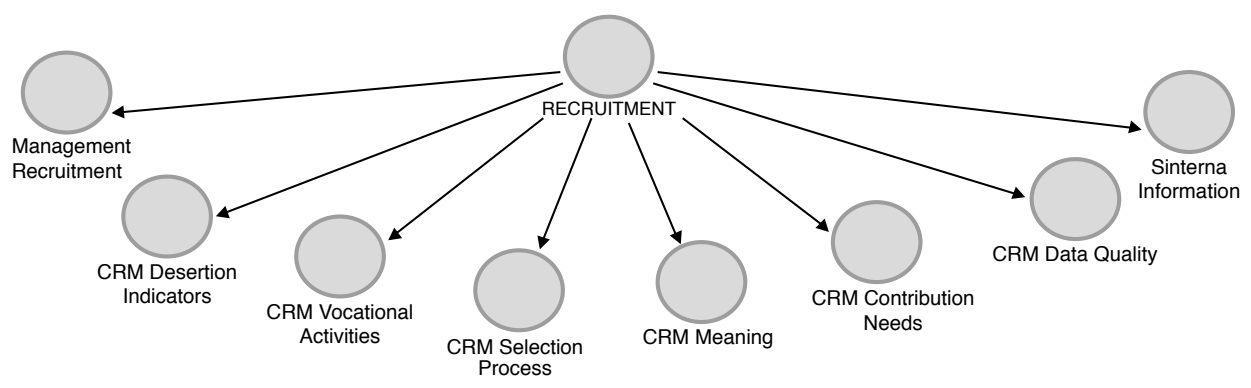

Figura 2. Nodo de Reclutamiento

Fuente: elaboración propia en NVivo 11 a partir de todos los discursos de los entrevistados

En la Figura 3, que representa el nodo gestión de matrícula, hay un factor importante y coincidente con la totalidad de las entrevistas que es la inclusión de poblaciones vulnerables, lo que hace que las IES diseñen planes de adaptación como se evidencia en las entrevistas. Lo anterior hace evidentes las prácticas de gestión para no permitir que ningún estudiante deserte.

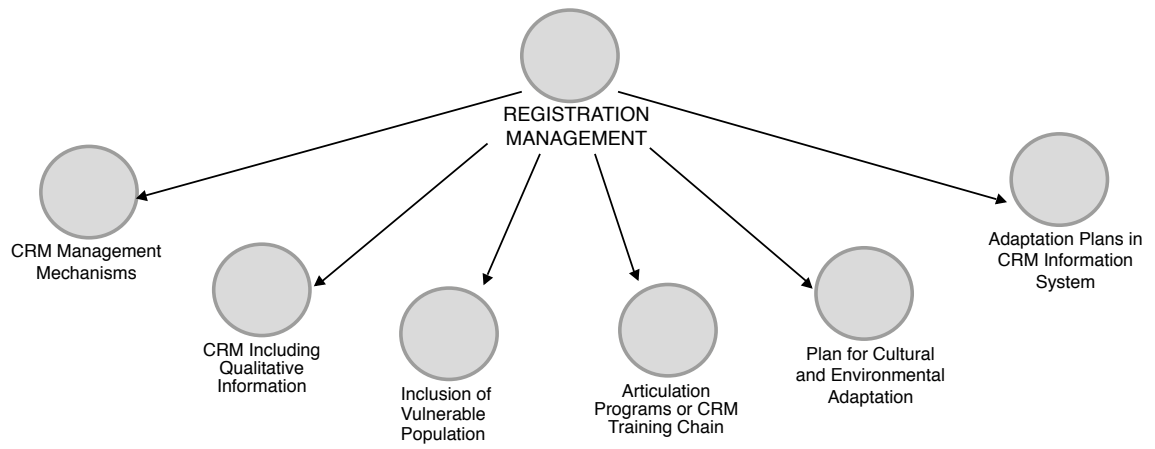

Figura 3. Nodo Gestión de Matrícula

Fuente: elaboración propia en NVivo 11 a partir de todos los discursos de los entrevistados

La Figura 4 representa el nodo graduación en el que las estrategias de graduación efectiva no dan evidencia de una articulación concreta con el CRM según la conexión que debería tener con el sector productivo y los procesos académicos que se desarrollan en la IES.

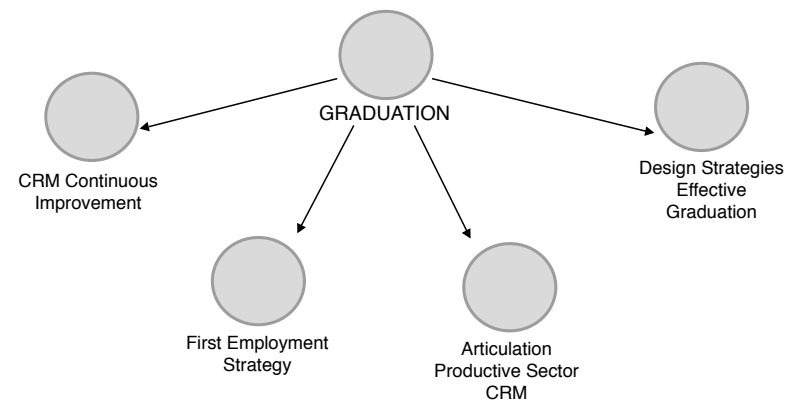

Figura 4. Nodo Graduación

Fuente: elaboración propia en NVivo 11 a partir de todos los discursos de los entrevistados 


\section{Discusión}

En la literatura sobre CRM en la educación superior se han identificado varios nodos dependientes dentro de la estructura y se evidenció que uno de los aspectos más relevantes y base de éste estudio es el significado que tiene el CRM para las instituciones, puesto que se basa en una herramienta de información y no en una filosofía, contrariamente a lo que indica Hernández-Mejía (2008).

Así mismo, ésta investigación complementa a Zeine et al (2015) quienes ubican a los estudiantes como centros del proceso como "cliente" cuando se cita al estudiante mencionando que son los primeros consumidores de las experiencias de aprendizaje ofrecidas por la institución educativa; los clientes y los consumidores son personas que evalúan y pagan los productos o servicios que se consideren beneficiosos, mientras que el distribuidor tiene como objetivo generar repeticiones de este proceso lo antes posible, así mismo indica que los estudiantes deben estar en el centro de las filosofías de servicio al cliente que se centran en la enseñanza y en los resultados del aprendizaje. Esto lo corroboran las entrevistas realizadas en donde se evidencia que el CRM "es un sistema de información estratégico que permite desarrollar la apuesta institucional de poner al estudiante en el centro de la acción institucional. Esto se evidencia cuando se afirma en esta investigación que con información se logra identificar, caracterizar y acompañar con estrategias de comunicación e intervención a todas las poblaciones de estudiantes” (Castellanos-Camargo, Rojas-Berrío, \& Robayo-Pinzón, 2016).

De la misma manera se anota que muchas actividades académicas, financieras, laborales, psicosociales y de inclusión, tanto de enrolamiento como de retención y persistencia de los estudiantes deben realizar las IES pues la mayoría de respuestas están en los apartados de estrategias académicas y financieras (Castellanos-Camargo et al., 2016).

Así mismo, y en concordancia con Bejou y Bejou (2012) cuando da cuenta del uso y apropiación del CRM para el reclutamiento; la gestión de la matrícula, la retención y la persistencia; la graduación y sus stakeholders, ya que según Nagori (2013), son los padres quienes pagan la educación de sus hijos y las empresas las que contratan egresados.

Como se menciona en el artículo "En relación con el estudio de la Gestión de Relaciones con el Cliente o CRM (en inglés CRM por Customer Relationship Management) y la educación superior hay muy poca documentación bibliográfica”.

Así se corroboró en esta investigación dado que gran parte de la bibliografía se encuentra en inglés y es aplicada a instituciones de Europa, Estados Unidos y Asia. En la mayoría de los casos se ve cómo el CRM está enfocado hacia los sistemas de información, antes que a una estratega institucional de conocimiento profundo del estudiante como cliente.

Como citan en el artículo digital e-marketers "Los vendedores digitales en América del Norte y Europa Occidental están utilizando diferentes tácticas para aumentar sus esfuerzos de marketing basados en datos. Se requieren ajustes en todos los NODOS identificados 
que son la base del proceso, desde el prospecto hasta el estudiante graduado, así mismo en la cultura institucional para poder apropiar y usar correcta y articuladamente el concepto de CRM”.

Otro hallazgo importante es que el seguimiento en plataformas tecnológicas se realiza desarticuladamente, las instituciones cuentan con diversos sistemas de información, casi que segmentados por áreas independientes. Como se detectó en varias de las instituciones entrevistadas, el manejo de diferentes sistemas de información hace que ésta sea fragmentada y no se dé realmente un seguimiento integral a los estudiantes y egresados de dichas instituciones. Como soporte de esta aseveración, en el cruce de información la mayoría de respuestas se ubicaron en la categorización del módulo emergente de seguimiento en plataforma, y el cruce con las estrategias de retención que tienen las instituciones escogidas para este estudio (Castellanos-Camargo et al., 2016). De aquí nace la necesidad sentida de integrar tanto la cultura organizacional como los sistemas de información, realizando interfaces desde las diferentes plataformas para poder tener control sobre los diversos ámbitos en que se desempeñan los estudiantes y maximizar por las acciones que conllevan a la retención de los estudiantes.

En el mismo cruce podemos evidenciar que tanto la estrategia académica como la financiera son las que tienen más incidencia en las instituciones; el tema laboral por su parte fue manifestad por una institución entrevistada como "En la actualidad, la razón por la que los estudiantes desertan en nuestra institución se relacionan con cuestiones económicas, falta de trabajo, disponibilidad horaria que acarrea el trabajar; la incertidumbre de la inserción laboral una vez recibidos; y en algunos casos aparecen razones de tipo "psicológico" o “emocional” (Castellanos-Camargo et al., 2016).

Sin embargo, esta investigación tuvo sus limitaciones. En primera instancia las instituciones dejaron ver que se tiene un uso limitado de la información, ya que al plasmarla en las entrevistas estas no se respondieron de manera rigurosa explicando a profundidad sus debilidades o fortalezas; como consecuencia de lo anterior el análisis de la información se realizó a través de las respuestas a los ítems incluidos en las entrevistas. De la misma manera, algunas de las instituciones adujeron que no podían contestar autónomamente y que debían llevarlo a las directivas para proceder a responderlo, sin embargo, dentro de las que contestaron la entrevista tenemos a varias de las más grandes y que han sido innovadoras, o están en proceso de innovación y crecimiento.

\section{Conclusiones}

Con base en el objetivo de esta investigación, se pretendía identificar si las IES en Colombia apropian el CRM para hacer un seguimiento real e integral a los estudiantes (clientes) y poder lograr la retención de los mismos. Como conclusiones tratamos de resolver las siguientes preguntas, planteadas en el documento:

¿Cuál es la relación existente entre el uso y apropiación del CRM y la permanencia del estudiante en el programa desde al ámbito de la Educación Superior? 
Como se evidencia en la investigación muchas de las causas de deserción en la institución tienen que ver con el área académica y económica, lo que nos lleva a proponer la implementación de estrategias de acompañamiento permanente y refuerzo en las áreas de conocimiento, integrando de alguna forma los sistemas de información académica con el sistema de admisiones, así mismo realizar un perfilamiento que permita que el mayor número de estudiantes tengan una actividad vocacional cuando son prospectos, es decir, cuando desde el colegio se les pueda guiar con toda la información pertinente al programa por el cual tienen preferencia.

¿El CRM se debe integrar a la función de marketing y ventas?: Como se evidenció en el estudio, es importante llamar la atención a este respecto, ya que la cultura del CRM no se debe basar solo en un sistema de información, en lo cual coincidieron la mayoría de las instituciones objeto del estudio; es importante que desde las directivas se impregne el CRM como una cultura organizacional y en la actualidad se evidencia que las IES están llenas de plataformas tecnológicas pero no integradas, lo cual permite que cada área sea autónoma, pero en muchas instituciones parece que las áreas fueran una empresa diferente. Las ventajas y oportunidades que tendrían las instituciones al ser rigurosas en la implementación real de un sistema CRM integrado, seguramente se verá reflejado en mejorar las tasas de retención de sus estudiantes.

¿El CRM se debe incluir dentro del Plan de desarrollo de las IES? Esta investigación nos da la base para recomendar a las IES que el marketing relacional se debe integrar a las tecnologías de información enfocándolas a los estudiantes como línea de base, a sus padres y a los proveedores de servicios educativos para realizar una labor integral.

¿Cómo integrar esta herramienta en la estrategia corporativa? La integración de sistemas de información a la estrategia se puede lograr realizando interfaces y haciendo una reingeniería de procesos e innovación tecnológica. Las instituciones entrevistadas, al tener unas la aprobación del Ministerio de Educación Nacional y otras el permiso de la Secretaría de Educación, han venido haciendo esfuerzos interesantes al respecto dentro de las quince áreas que se califican para aseguramiento de la calidad de las IES

Como conclusión general, podemos tomar este concepto como base para desarrollar estudios a las instituciones, de acuerdo con su carácter. Al respecto debemos diseñar un programa de actividades encaminadas al relacionamiento con nuestros clientes que nos permita personalizar a cada uno de nuestros estudiantes y poder fortalecer el reclutamiento, la gestión de matrícula, la retención, la persistencia y la graduación, identificados como NODOS principales en esta investigación, en los cuales se puede realizar un indeterminado número de actividades que permitan aumentar la tasa de retención de las IES.

Es relevante indicar que el instrumento aplicado a las IES es muy sensible, por cuanto cada una señaló que sí se están haciendo actividades, lo cual es cierto, pero no como una política institucional sino como el enfoque de cada una de las áreas, que finalmente pueden apuntar hacia el mismo objetivo, pero si estuvieran articulados los resultados serían mejores. 


\section{Conflicto de intereses}

Los autores declaran no tener ningún conflicto de intereses

\section{Referencias bibliográficas}

1. Abubakar, M. M., \& Mokhtar, S. S. M. (2015). Relationship marketing, long term orientation and customer loyalty in higher education. Mediterranean Journal of Social Sciences, 6(4), 466-474. https://doi.org/10.5901/mjss.2015.v6n4p466

2. Bejou D., Bejou A. (2012). Shared Governance and Punctuated Equilibrium in Higher Education: The Case for Student Recruitment, Retention, and Graduation.

3. Castellanos-Camargo, L. G., Rojas-Berrío, S. P., \& Robayo-Pinzón, Ó. J. (2016). Entrevistas de la Investigación. Bogotá D.C., Colombia.

4. Daradoumis, T., Faulin, J., Juan, A., Martínez-López, F., Rodríguez-Ardura, I., \& Xhafa, F. (2008). Expanding the customer relationship management scope to the non-profit organizations: an analisys focused on the E-University Domain. In M. Baptista-Nunes, P. Isaías, \& P. Powell (Eds.), IADIS International Conference e-Commerce 2008 (pp. 113119). Algarve, Portugal: International Association for Development of the Information Society. Retrieved from http://www.iadisportal.org/is-2008-proceedings

5. Dužević, I., \& Čeh Časni, A. (2015). Student and faculty perceptions of service quality: the moderating role of the institutional aspects. Higher Education, 70(3), 567-584. https://doi.org/10.1007/s10734-014-9857-3

6. Frow, P., Payne, A., Wilkinson, I., \& Young, L. (2015). CRM and customer management: Identifying and confronting dark side behaviours. In The Dark Side of CRM: Customers, Relationships and Management (pp. 21-38). University of Sydney Business School, Australia. https://doi.org/10.4324/9781315753737

7. Gil-Lafuente, A., \& Luis-Bassa, C. (2011). La Innovación centrada en el cliente utilizando el Modelo de Inferencias en una Estrategia CRM. Investigaciones Europeas de Dirección Y Economía de La Empresa, 17(2), 15-32.

8. Hernández-Mejía, M.-C. (2008). La cultura orientada al cliente trasciende a una estructura de CRM en las instituciones de educación superior públicas y privadas (universidades) del Eje Cafetero. Universidad Nacional de Colombia. Retrieved from http://www.bdigital.unal.edu.co/1128/1/marthaceciliahernandezmejia.2008.pdf

9. Khashab, B., Gulliver, S. R., Alsoud, A., \& Kyritsis, M. (2014). Scoping customer relationship management strategy in HEI understanding steps towards alignment of customer and management needs. In ICEIS 2014 - Proceedings of the 16th International Conference on Enterprise Information Systems (Vol. 2, pp. 267-274). School of Business Informatics, Systems and Accounting, University of Reading (UK), Henley Business School, RG6 6UR, Reading, Berkshire, United Kingdom. Retrieved from https://www. scopus.com/inward/record.uri?eid=2-s2.0-84902344163\&partnerID=40\&md5=3d76d5 4eac5484ea58b2607eebcb56d5

10. Kornbluh, M. (2015). Combatting Challenges to Establishing Trustworthiness in Qualitative Research. Qualitative Research in Psychology, 12(4), 397-414. https://doi. org/10.1080/14780887.2015.1021941

11. Montoya-Restrepo, A., Montoya-Restrepo, I., \& Rojas-Berrio, S. (2012). Percepción de la calidad y satisfacción de la elección de los estudiantes de primer semestre en la 
Facultad de Ciencias Económicas de la Universidad Nacional de Colombia. Poliantea, 8(14), 71-94.

12. Nagori, V. (2013). CRM for academic institution and universities. In Enterprise Resource Planning: Concepts, Methodologies, Tools, and Applications (Vol. 1-3, pp. 198-204). https://doi.org/10.4018/978-1-4666-4153-2.ch012

13. Pember, E. R., Owens, A., \& Yaghi, S. (2014). Customer relationship management: A case study from a metropolitan campus of a regional university. Journal of Higher Education Policy and Management, 36(2), 117-128. https://doi.org/10.1080/136008 0X.2013.861056

14. Petrella, C. (2008). Gestión de la relación de las universidades con docentes, estudiantes y egresados. Revista Iberoamericana de Educacion, 47(5), 1-14.

15. Rowley, J. (2003). Retention: Rhetoric or realistic agendas for the future of higher education. International Journal of Educational Management, 17(6), 248-253. https:// doi.org/10.1108/09513540310487578

16. Sahney, S., Banwet, D. K., \& Karunes, S. (2008). An integrated framework of indices for quality management in education: A faculty perspective. TQM Journal, 20(5), 502-519. https://doi.org/10.1108/17542730810898467

17. Santamaría, F.-A., \& Bustos, A. (2013). Permanencia y abandono en la educación superior: una experiencia de investigación a partir de las voces de los jóvenes estudiantes. Revista Infancias Imágenes, 12(2), 73-80.

18. Seeman, E. D., \& O'Hara, M. (2006). Customer relationship management in higher education: Using information systems to improve the student-school relationship. Campus-Wide Information Systems, 23(1), 24-34. https://doi. org/10.1108/10650740610639714

19. Zeine, R., Palatnick, F., Boglarsky, C., Blessinger, P., Herrick, B., \& Hamlet, M. (2015). Customer service focus and Mission Articulation as measures of organizational effectiveness in higher education institutions: Driving student success. Management Education, 14(1), 1-10. 